\title{
Study on Method of Underwater Electromagnetic Target Dimension Recognition
}

\author{
Zhiliang Ren, Siwei Tan, Changcun Sun \\ Department of Weaponry Engineering \\ Naval University of Engineering \\ Wuhan China \\ tsw_0_0_0@163.com
}

\author{
Jiong Sun \\ Office of Research \& Development \\ Naval University of Engineering \\ Wuhan China \\ Jiongsun@163.com
}

\begin{abstract}
The detection range and the equipment-target encounter speed are key factors to the capability of target dimension recognition in an underwater electromagnetic detection system. In view of that, a novel dimension recognition method based on the detection range and the encounter speed extracting was proposed in this paper. This method is able to suppress interference more effectively and improve the capability of the target dimension recognition in the underwater electromagnetic detection system. The target dimension features were analyzed. The extracting algorithms for the detection range and the encounter speed were deduced. The feasibility of improving the target dimension recognition capability by considering factors such as the detection range and the encounter speed was proved via examples.
\end{abstract}

Keywords-electromagnetic detection; underwater electromagnetic propagation; feature extracting; dimension recognition

\section{INTRODUCTION}

The target recognition capability is very important to the underwater electromagnetic detection equipment. However, the energy attenuation in electromagnetic wave propagation process underwater is serious and strongly nonlinear. As a result, the system operating frequency, the signal waveform and the system detection range are more likely to be restricted. Only the signal strength and delay of the electromagnetic wave can be utilized in the underwater target detection system design. If the targets' shape, size, equipment-target encounter distance and speed differ considerably, the accuracy and consistence of the target detection would be affected. Then the certain action control requirement could not be realized easily and reliably.

Recognition by the underwater targets' dimension features can not only improve the capabilities for target detection and recognition in the electromagnetic detection system, but also effectively suppress interference and reject false targets with low similarity or weak relevance to the true ones. So the proposed method has great significance to improve the operation reliability and recognition accuracy of the system.

\section{DIMENSION ELECTROMAGNETIC FEATURES OF UNDERWATER TARGET}

For the active electromagnetic detection equipments, the target reflected electromagnetic field signal is a kind of amplitude modulation waveform modulated by $H_{z m} \cdot f(t)$ [1].

$$
\begin{aligned}
& H_{z p}(t)=H_{z m} \cdot f(t) \cdot \sin \left(\omega_{0} t+\varphi_{0}\right) \\
& f(t)=e^{-\rho t^{2}} \\
& \rho=\frac{0.36 V_{T K}^{2} \sin ^{2} \theta_{k}}{B_{k}^{2}+(1+0.063 r)} \\
& B_{k}=K_{B} \frac{B}{\sin \theta_{k}}
\end{aligned}
$$

In the equations above, we defined the $H_{z m}$ as maximum magnitude of the received signal $(\mathrm{A} / \mathrm{m})$; the $\omega_{0}$ as signal frequency (rad); the $\varphi_{0}$ as initial phase of the received signal (rad); the $V_{T K}$ as relative velocity $(\mathrm{m} / \mathrm{S})$; the $r$ as detection range (m); the $\theta_{k}$ as encounter angle (rad); the $B_{k}$ as target equivalent width $(\mathrm{m})$; the $K_{B}$ as target shape factor; the $B$ as target actual width (m).

From (1) to (4), the target reflected signal waveform is drawn as shown in Fig.1. The target dimension $B$ could be estimated from the signal duration time $\Delta T$. The features of the underwater target electromagnetic dimension could be described as:

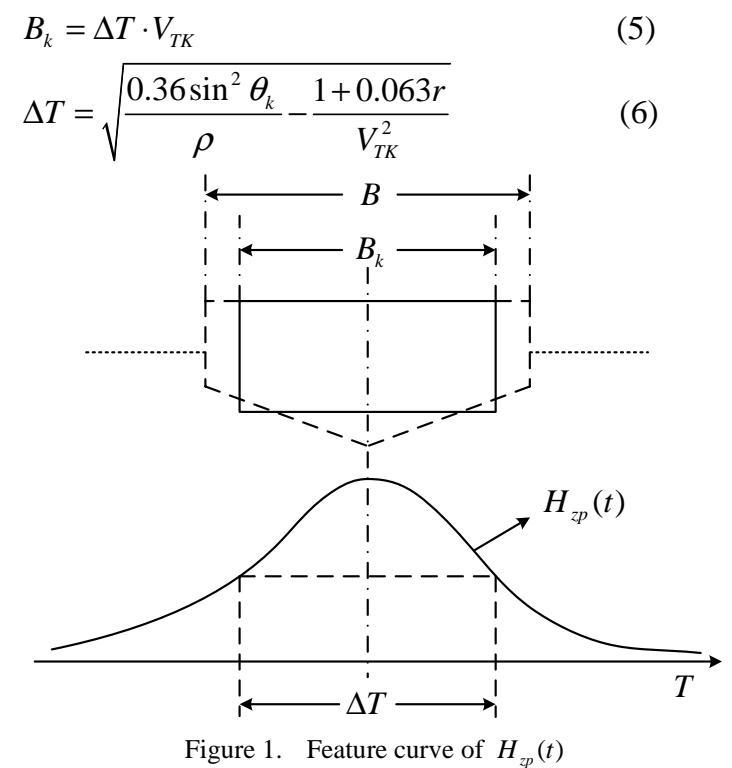

From (5), (6) and Fig.1 we can see that, the target dimension recognition could be realized easily by testing the 
signal duration time $\Delta T$ in condition of a known target and encounter situation. Based on that method, the conventional electromagnetic detection equipment usually sets a value $\Delta T$ to judge the target recognition and locate it for a further action. However, in a practical application, the target dimension factors $\left(B 、 K_{B}\right)$, encounter conditions $\left(V_{T K}\right.$ 、

$\left.\theta_{k}\right)$ and system capability $(r)$ are usually unknown and nonlinear. If the dimension factors mentioned above are heterogeneous and could not be achieved beforehand, the conventional target dimension recognition method would cause a serious deviation. As a result of that, the optimal action time controlling would fail. Even an omission or a misoperation would happen [2-4].

\section{TARGET DIMENSION FACTORS}

In the process of the system and the target encountering, there are many objective factors influencing the electromagnetic wave receiving and the target dimension recognition, such as target shape factor $K_{B}$, encounter angle $\theta_{k}$, and so on. Those factors can be given as experience values, and would not cause a serious deviation in dimension recognition. But the encounter speed $V_{T K}$ and detection range $r$ are more critical to the dimension recognition as shown in Fig.2. Considering certain electromagnetic detection equipment and a known target, we assume that the effective received signal duration time is $\Delta T_{A}$ in a situation of a longer equipment-target encounter distance and a higher relative velocity. Accordingly, the effective received signal duration time is $\Delta T_{B}$ in a condition of a shorter encountering distance and a lower relative velocity. Obviously, $\Delta T_{A}$ and $\Delta T_{B}$ are different even for the same target. If the system still recognizes the target by judging the invariable duration time set beforehand, there must be a serious mistake in the target dimension recognition [5].

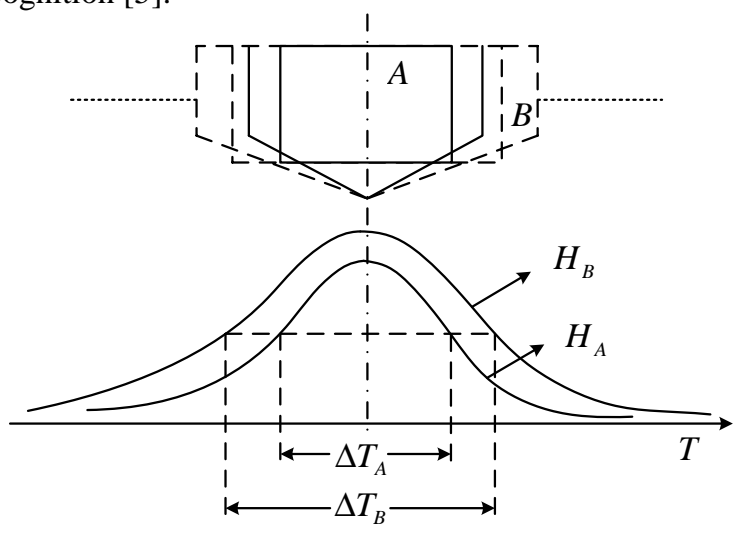

Figure 2. $\quad r$ 's and $V_{T K}$ 's influences on $\Delta T$

Considering the calculation of encounter speed $V_{T K}$ and detection range $r$, it is very convenience to achieve the values via Doppler velocimetry and pulse ranging in the air. However, the underwater electromagnetic wave propagation features limit the detection system operation parameters. If the overall platform is taken into consideration and an improvement is obtained in the system redesign, the value of encounter speed $V_{T K}$ could be provided by a self-guided system. But it is more favorable for the detection system to solve that problem. As we known, the self-guided system is more likely to be blind in the near range of the target. But the underwater electromagnetic wave only can be utilized in near field. As a result, the parameter $r$ could not be provided by the self-guided system in practical application.

A. Parameter $r$ Acquisition

According to the propagation features ${ }^{[1]}$ of the electromagnetic wave in a conductive media, the magnitude of the electromagnetic wave will exponential decay and the phase lag will increase as the propagation distance increases. We define the magnitude attenuation coefficient as $K_{r}$ and the phase shift coefficient as $\Phi_{r}$ :

$$
\begin{aligned}
& K_{r}=\frac{|E|}{E_{0}}=\frac{|H|}{H_{0}}=e^{-\beta r} \\
& \Phi_{r}=-\alpha r
\end{aligned}
$$

In (7) and (8), $\alpha$ and $\beta$ are defined as follows:

$$
\begin{aligned}
& \alpha=\omega \sqrt{\frac{\mu \varepsilon}{2}}\left[\sqrt{1+\frac{\sigma^{2}}{\omega^{2} \varepsilon^{2}}}+1\right]^{\frac{1}{2}} \\
& \beta=\omega \sqrt{\frac{\mu \varepsilon}{2}}\left[\sqrt{1+\frac{\sigma^{2}}{\omega^{2} \varepsilon^{2}}}-1\right]^{\frac{1}{2}}
\end{aligned}
$$

The $\varepsilon, \mu$ and $\sigma$ are defined as basic parameters describing the media electromagnetic features ${ }^{[1]}$. The $\alpha$ and $\beta$ are respectively defined as phase shift constant and magnitude attenuation constant $[6,7]$.

Though the features of magnitude attenuation and phase shift in underwater electromagnetic wave propagation are nonlinear, the value of propagation distance still can be obtained from the features mentioned above. Comparing the methods of distance extracting from magnitude to the method from phase shift, the method of ranging from the propagation phase shift is better in stability and accuracy [8]. B. Parameter $V_{T K}$ Acquisition

According to the parameter $V_{T K}$ from the received signal $f(t)$ or $H_{z p}(t)$, we could derivate the $\rho$ calculation equations as follows in condition of sampling at equal time intervals $\Delta t$, as shown in Fig.3. It can be realized in the electromagnetic detection system design.

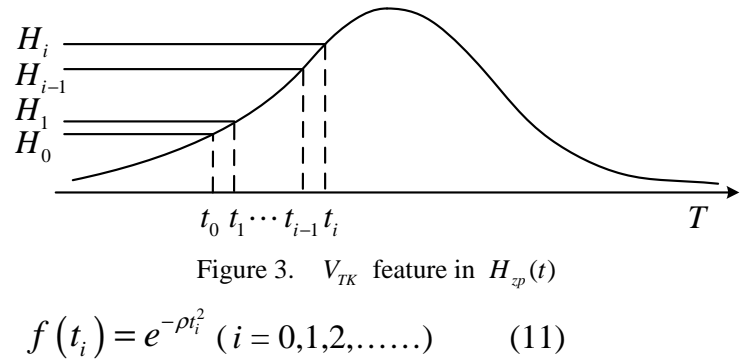

After processing the sample data, the further calculations are as follows: 


$$
\begin{aligned}
& \ln \frac{f\left(t_{i-1}\right)}{f\left(t_{i}\right)}=\rho\left(t_{i}-t_{i-1}\right)\left(t_{i}+t_{i-1}\right) \\
& =\rho \cdot \Delta t\left(t_{i}+t_{i-1}\right) \\
& \ln \frac{f\left(t_{i-1}\right)}{f\left(t_{i+1}\right)}=\rho\left(t_{i+1}-t_{i-1}\right)\left(t_{i+1}+t_{i-1}\right) \\
& =\rho \cdot 2 \Delta t\left(t_{i+1}+t_{i-1}\right) \\
& \rho=\frac{B-A}{t_{i+1}-t_{i}}=\frac{B-A}{\Delta t}
\end{aligned}
$$

In (14), the variable $A$ and $B$ are defined as follows:

$$
A=\frac{1}{\Delta t} \ln \frac{f\left(t_{i-1}\right)}{f\left(t_{i}\right)} ; \quad B=\frac{1}{2 \Delta t} \ln \frac{f\left(t_{i-1}\right)}{f\left(t_{i+1}\right)}
$$

The target dimension recognition in uncertain conditions could be realized by using (5) and (6) according to the dimension factors $[9,10]$.

\section{EXAMPLES ANALYSIS}

\section{A. Example $A$}

In this example we assumed that the target shape and the encounter speed $V_{T K}$ had been known beforehand, and discussed the dimension estimation in condition of different detection range

$\left(0.5 m \leq r \leq 6 m, r_{i+1}=r_{i}+\Delta r, i=0,1, \ldots \ldots, \Delta r=0.5 m\right)$.

As we known, the encounter situation changes randomly as the underwater electromagnetic detection equipment passing through the target. This uncertain situation varies the value of target signal duration time $\Delta T$ and makes the problem of target dimension recognition more complex. In view of that, this paper defined the vertical encounter angle $\varphi_{T K}$, as shown in Fig.4, as the angle between the equipment voyage direction and the equipment-target connection as the equipment detected the target for the first time as getting close to it. Considering the most adverse encounter condition, as the detection equipment passing through the bottom of a cylindrical target in an abeam direction, the corresponding relationships between the distance traveled or the signal duration time and the value of $\varphi_{T K}$ are shown in Fig.4.

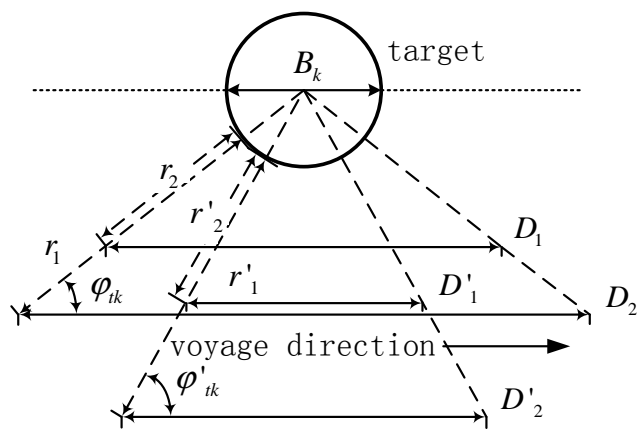

Figure 4. The sketch map of encounter situation

In order to estimate the target dimension when the $r$ and the $\varphi_{T K}$ vary, this paper assumed that the value of the vertical encounter angle $\varphi_{T K}$ varied in $75^{\circ}, 60^{\circ}, 45^{\circ}, 30^{\circ}$, $15^{\circ}$ and $0^{\circ}$. In addition the signal traveled journey $D$ should be described as the equation below:

$$
D=2 *\left(r+\frac{B_{k}}{2}\right) * \sin \left(\frac{\varphi_{t k}}{2}\right)
$$

This paper used the signal duration time $\Delta T$ to reflect the dimension estimation in (16):

$$
\Delta T=\frac{D}{V_{T K}}
$$

The simulation results were shown in the Fig.5.

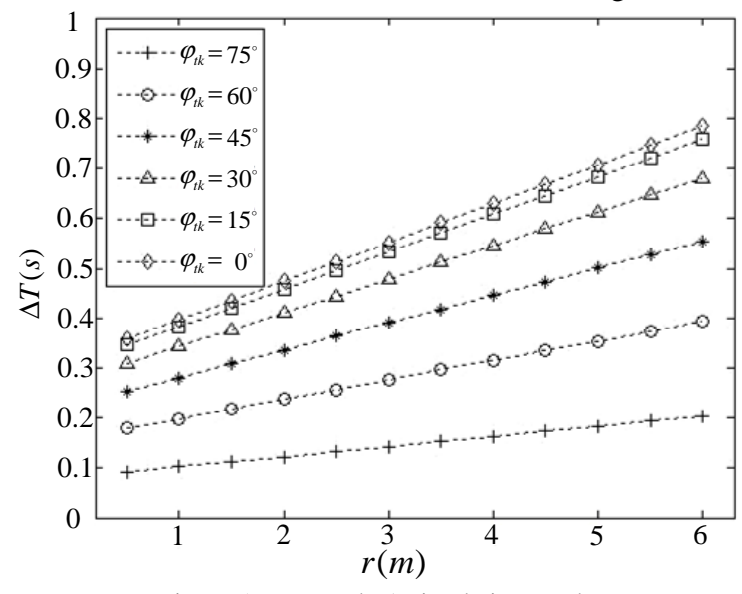

Figure 5. Example A simulation results

\section{B. Example B}

In this example we assumed that the target shape and the detection range $r(5 \mathrm{~m})$ had been known beforehand, and discussed the dimension estimation in condition of different encounter speed $V_{T K}$ (20 kn $\left.\leq r \leq 50 \mathrm{kn}, V_{i+1}=V_{i}+\Delta V, i=0,1, \ldots \ldots, \Delta V=5 \mathrm{kn}\right)$.

The dimension estimation results could be calculated by (16) when the traveled journey was a constant as shown in Fig.6.

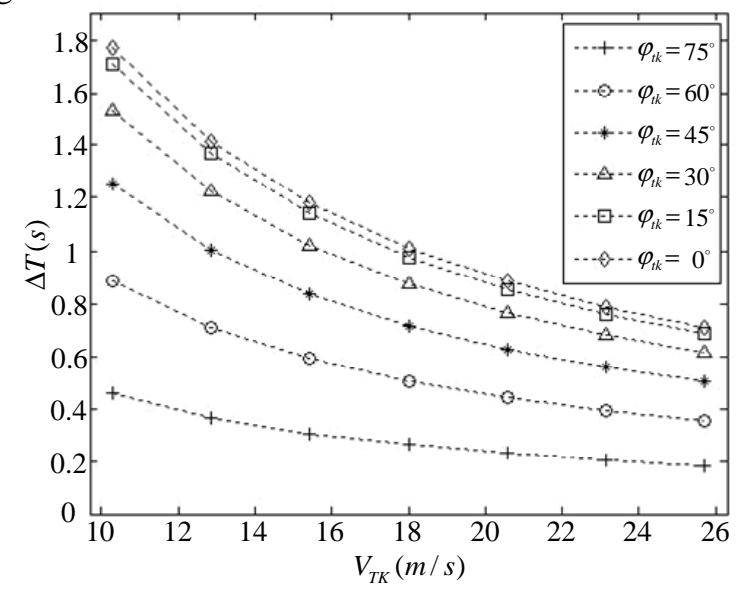

Figure 6. Example B simulation results

From the simulation results, we could draw the conclusions that the value of signal duration time $\Delta T$ varies as the detection range $r$, the encounter speed $V_{T K}$ and the vertical encounter angle $\varphi_{T K}$ varying. When $V_{T K}$ is a 
constant, the $\Delta T$ increases linearly as the $r$ increasing. In addition, the smaller $\varphi_{T K}$ the longer $\Delta T$, and the $\Delta T$ increases faster. When $r$ is a constant, the $\Delta T$ decreases nonlinearly as the $V_{T K}$ increasing. The smaller $\varphi_{T K}$ the longer $\Delta T$, and the $\Delta T$ decreases rapidly.

\section{CONCLUSIONS}

The system detection rang and the equipment-target encounter speed are the key factors to the dimension recognition in underwater electromagnetic target detection. Those parameters affect the target dimension calculation and judgment directly. Therefore, the method, proposed in this paper, that recognize the target dimension by extracting the parameters $r$ and $\Delta T$ in real-time is able to improve the capability of target dimension recognition in underwater electromagnetic detection system. It achieves the purpose of eliminating the interference from the false target and suppresses interference effectively.

\section{REFERENCES}

[1] Shaoqing Wang, Jianmin Liu, "Torpedo proximity fuze principle and design,” Xi'an, China: Northwestern Polytechnical University Press, 1992.

[2] Yuzheng Yuan, "Electromagnetic distance measurement elements," Beijing, China: National Defense Indusrty Press, 1990.
[3] John J. Holmes, "Reduction of a ship’s magnetic field signatures," Synthesis Lectures on Computional Electromagnetic, vol.23, pp.1-76, January, 2008.

[4] Earl G. Williams, Nicolas P. Valdivia, "Near-field electromagnetic holography in conductive media,” IEEE Transaction on Antennas and Propagation, vol.58, pp.1181-1192, April, 2010.

[5] Zhiliang Ren, Jun Du, "The study on ranging torpedo hitting,” Journal of Detection \& Control, vol.25, pp.6-8, June, 2003.

[6] Mingzhou Wang, Chongyang Hao, Xiaowen Huang, "Study on underwater target dimension recognition based on bearings analysis of signal correlation feature,” Journal of Northwestern Polytechnical University, vol.21, pp.317-320, June, 2003.

[7] Mingzhou Wang, Chongyang Hao, Xiaowen Huang, “Target dimension recognition based on short time cross-spectrum by using splitting beam array,” Journal of Data Acquision \& Processing, vol.18, pp.440-443, December, 2003.

[8] Yuchuan Yang, Huailin Cui, Zhishun Li, "Research on the simulation method of an underwater target dimension characteristic based on circular arc array,” Acta Acustica, vol.30, pp.558-565, November, 2005.

[9] Jin Liu, Hongbing Ji, "An improved algorithm for IR object recognition,” Pattern Recognition and Artificial Intelligence, vol.23, pp.115-119, February, 2010.

[10] Zhaohui Liu, Zhanping Fu, Mingzhou Wang, "Underwater target identification based on the methods of bearing and cross-spectrum," Acta Armamentarii, vol.27, pp.932-935, September, 2006. 